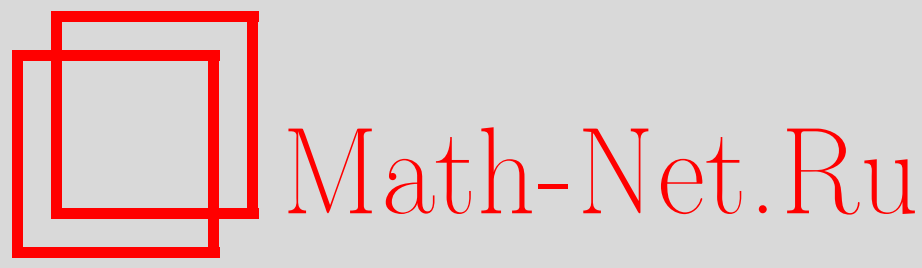

В. В. Цегельник, Гамильтонианы, ассоциированные с третьим и пятым уравнениями Пенлеве, ТМФ, 2010, том 162, номер 1, 69-74

DOI: https://doi.org/10.4213/tmf6455

Использование Общероссийского математического портала Math-Net.Ru подразумевает, что вы прочитали и согласны с пользовательским соглашением http://www . mathnet.ru/rus/agreement

Параметры загрузки:

IP: 3.82 .47 .9

26 апреля 2023 г., 12:41:26

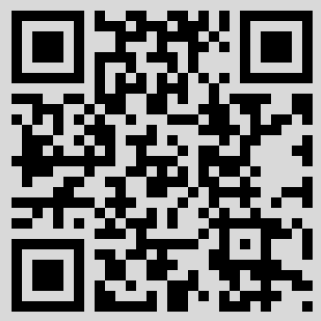




\section{ГАМИЛЬТОНИАНЫ, АССОЦИИРОВАННЫЕ С ТРЕТЬИМ И ПЯТЫМ УРАВНЕНИЯМИ ПЕНЛЕВЕ}

Получено дифференциальное уравнение типа Пенлеве для простейшего рационального гамильтониана, ассоциированного с пятым уравнением Пенлеве в случае $\gamma \neq 0, \delta=0$. Доказано существование ассоциированных с пятым уравнением Пенлеве в случае $\gamma \neq 0, \delta=0$ гамильтонианов нерационального типа. Получено обобщение формул Гарнье и Окамото рациональных гамильтонианов, ассоциированных с третьим уравнением Пенлеве.

Ключевые слова: третье уравнение Пенлеве, пятое уравнение Пенлеве, гамильтониан.

\section{1. ВВЕДЕНИЕ}

В последние три десятилетия наблюдается заметный рост интереса к исследованию определенных классов непрерывных и дискретных вероятностных моделей, известных под названием "модели случайно-матричного типа". Источники таких моделей весьма разнообразны [1].

Одной из наиболее важных характеристик указанных моделей является нуль-вероятность - вероятность отсутствия частиц в заданном интервале или объединении интервалов.

Нуль-вероятности, как правило, могут быть представлены в виде определителей Фредгольма $\left.\operatorname{det}(\mathbf{1}-K)\right|_{J}$, где $K$ есть некоторый интегральный оператор, а $J-$ множество, где не должно быть частиц. Ядро оператора $K$ обычно имеет вид

$$
K(x, y)=\frac{A(x) B(y)-B(x) A(y)}{x-y} \sqrt{\psi(x) \psi(y)}
$$

с подходящими функциями $\psi, A, B$. Единственный известный на настоящий момент способ вычисления таких определителей Фредгольма состоит в их характеризации как решений некоторого обыкновенного дифференциального уравнения или системы уравнений с частными производными. В работе [2] решена задача о выводе дифференциального уравнения для нуль-вероятностей в случае синус-ядра, которое имеет вид (1) с $\psi(x)=1 / x, A(x)=\sin x, B(x)=\cos x$, и показано, что значение определителя Фредгольма единичного оператора минус синус-ядро, суженое на интервал

*Белорусский государственный университет информатики и радиоэлектроники, Минск, Беларусь. E-mail: tsegvv@bsuir.by 
переменной длины $t$, выражается через решение обыкновенного дифференциального уравнения

$$
\left(t \frac{d^{2} \sigma}{d t^{2}}\right)^{2}=-4\left(t \frac{d \sigma}{d t}-1-\sigma\right)\left[t \frac{d \sigma}{d t}+\left(\frac{d \sigma}{d t}\right)^{2}-\sigma\right]
$$

Последовательными преобразованиями $\sigma(t)=t d \rho(t) / d t$,

$$
\rho(t)=\rho_{0} \int_{0}^{t}\left\{\frac{\tau}{4 w\left(1-w^{2}\right)}\left[\left(\frac{d w}{d \tau}\right)^{2}+4 w^{2}\right]-\frac{(1+w)^{2}}{4 \tau w}\right\} d \tau, \quad \rho_{0}=\text { const },
$$

функция $\sigma(t)$ выражается через решение пятого уравнения Пенлеве $\left(P_{5}\right)$

$$
\frac{d^{2} w}{d t^{2}}=\frac{3 w-1}{2 w(w-1)}\left(\frac{d w}{d t}\right)^{2}-\frac{1}{t} \frac{d w}{d t}+\frac{(w-1)^{2}}{t^{2}}\left(\alpha w+\frac{\beta}{w}\right)+\frac{\gamma w}{t}+\frac{\delta w(w+1)}{w-1}
$$

при значениях параметров $\alpha=-\beta=1 / 2, \gamma=-2 i, \delta=2$.

Следует отметить, что впервые связь между определителями Фредгольма интегральных операторов и решениями уравнений Пенлеве была установлена в работе [3]. Точнее, авторами этой работы было показано, что однопараметрическое семейство решений уравнения

$$
\frac{d^{2} \varphi}{d \tau^{2}}+\frac{1}{\tau} \frac{d \varphi}{d \tau}=\frac{1}{2} \operatorname{sh} 2 \varphi+2 a_{0} \tau^{-1} \operatorname{sh} \varphi
$$

где $a_{0}$ - параметр, являющегося частным случаем третьего уравнения Пенлеве $\left(P_{3}\right)$

$$
\frac{d^{2} \lambda}{d \tau^{2}}=\frac{1}{\lambda}\left(\frac{d \lambda}{d \tau}\right)^{2}-\frac{1}{\tau} \frac{d \lambda}{d \tau}+\frac{1}{\tau}\left(a \lambda^{2}+b\right)+c \lambda^{3}+\frac{d}{\lambda}
$$

с параметрами $a, b, c, d$, выражается в терминах определителей Фредгольма специального типа.

Работа [3] в идейном плане тесно связана с работами [4]-[6], в которых решалась классическая проблема (берущая начало с работы [7]) вычисления спиновых корреляционных функций двумерной модели Изинга. В работах [4]-[6] был получен важный результат [8]: скейлинговый предел двухточечной корреляционной функции двумерной модели Изинга допускает замкнутое выражение через решение уравнения $P_{3}$ при $a=b=0, c=-d=1$. Характерно [9], что главная часть этого выражения содержит гамильтониан

$$
K_{0}=\frac{1}{\tau}\left(\lambda^{2} \mu^{2}-3 \lambda \mu-\frac{\tau^{2}}{4 \lambda^{2}}-\frac{1}{4} \tau^{2} \lambda^{2}+\frac{9}{4}+\frac{\tau^{2}}{2}\right),
$$

ассоциированный с уравнением $\left(P_{3}\right)$ в случае $a=b=0, c=-d=1$.

В работе [10] получено обобщение уравнения (2) на случай произвольных параметров уравнения $P_{5}$ таких, что $\gamma \delta \neq 0$. Для построения указанного уравнения автор работы [10] использовал хорошо известный факт [11] представления уравнения $P_{5}$ в виде системы Гамильтона

$$
w^{\prime}=\frac{\partial H}{\partial u}, \quad u^{\prime}=-\frac{\partial H}{\partial w},
$$


где

$$
\begin{aligned}
H= & \frac{1}{t}\left\{w(w-1)^{2} u^{2}-\left[\theta_{0}(w-1)^{2}-\theta_{1} w(w-1)-\eta_{1} t w\right] u+\right. \\
& \left.+\frac{1}{4}\left[\left(\theta_{0}+\theta_{1}\right)^{2}-\theta_{\infty}^{2}\right](w-1)\right\}, \\
\alpha= & \frac{1}{2} \theta_{\infty}^{2}, \quad \beta=-\frac{1}{2} \theta_{0}^{2}, \quad \gamma=-\eta_{1}\left(\theta_{1}+1\right), \quad \delta=-\frac{1}{2} \eta_{1}^{2} .
\end{aligned}
$$

Обозначая независимую переменную $t$ через $z$, уравнение $P_{5}$ в случае $\gamma \neq 0, \delta=0$ будем рассматривать в виде

$$
\frac{d^{2} w}{d z^{2}}=\frac{3 w-1}{2 w(w-1)}\left(\frac{d w}{d z}\right)^{2}-\frac{1}{z} \frac{d w}{d z}+\frac{(w-1)^{2}}{z^{2}}\left(\alpha+\frac{\beta}{w}\right)+\frac{r w}{z},
$$

где $r \neq 0$ - произвольная постоянная.

Известно [12], что уравнение $P_{5}$ представимо в виде системы (4) с гамильтонианом

$$
\begin{gathered}
H_{1}=\frac{1}{z}\left[w(w-1)^{2} u^{2}+m(w-1)^{2} u+n(w-1) w u+\frac{r z}{2(w-1)}\right], \\
m^{2}+2 \beta=0, \quad(m+n)^{2}-2 \alpha=0 .
\end{gathered}
$$

Целью настоящей работы является построение дифференциального уравнения, определяющего функцию $h(z)=z H_{1}(z, u(z), w(z))$, которое, следуя работе [13], будем называть $h$-уравнением. Мы покажем, что указанное $h$-уравнение обобщает хорошо известное уравнение, полученное в работах [14], [15] при исследовании моделей случайно-матричного типа. Будет получено обобщение формулы (6) и тем самым доказано существование ассоциированных с уравнением (5) гамильтонианов нерационального типа.

Будет получена формула гамильтониана, ассоциированного с уравнением $P_{3}$, являющаяся обобщением выражения (3) и формулы Гарнье [16] гамильтониана

$$
K_{1}=\frac{1}{\tau}\left(2 \lambda^{2} \mu^{2}-3 \lambda \mu-\frac{a}{4} \tau \lambda+\frac{b \tau}{4 \lambda}-\frac{c \tau^{2} \lambda^{2}}{8}+\frac{d \tau^{2}}{8 \lambda^{2}}\right),
$$

также ассоциированного с $P_{3}$.

\section{2. УРАВНЕНИЕ (5) И АССОЦИИРОВАННЫЕ С НИМ ГАМИЛЬТОНИАНЫ}

Введем в рассмотрение функцию $h(z)=z H_{1}(z, u(z), w(z))$, заданную на решениях системы (4) с гамильтонианом $H_{1}$. Тогда справедливы следующие соотношения:

$$
\begin{gathered}
w=\frac{2 h^{\prime}+r}{2 h^{\prime}} \\
h=\frac{z^{2} w^{2}-\left[m(w-1)^{2}+n w(w-1)\right]^{2}}{4 w(w-1)^{2}}+\frac{r z}{2(w-1)} .
\end{gathered}
$$


Действительно, формула (8.1) получается, если продифференцировать обе части равенства $h(z)=z H_{1}(z, u(z), w(z))$ и из полученного соотношения найти функцию $w$. Соотношение (8.2) можно получить, если в формуле (6) выразить переменную $u$ через $w, w^{\prime}$ согласно уравнениям Гамильтона. Подстановка функции $w$ (заданной формулой (8.1)), а также ее производной $w^{\prime}=-r h^{\prime \prime} /\left(2 h^{\prime 2}\right)$ в соотношение (8.2) позволяет получить уравнение, определяющее функцию $h$ :

$$
z^{2} h^{\prime \prime 2}+4 h^{\prime 2}\left(z h^{\prime}-h\right)+h^{\prime}\left[\left(2 r z-n^{2}\right) h^{\prime}-2 r h-(m+n) n r\right]-\frac{(m+n)^{2} r^{2}}{4}=0 .
$$

Формулы (8.1) и (8.2), определяющие взаимно однозначное соответствие между решениями уравнений (5) и (9), позволяют сделать вывод о том, что уравнение (9) является уравнением типа Пенлеве (Р-типа). Действительно, с одной стороны, общее решение уравнения (5) не имеет подвижных критических особых точек. С другой стороны, функция $h$ (решение уравнения (9)) в силу формулы (8.2) рациональным образом выражается через решение уравнения (5) и его производную.

Пример. Уравнение (5) при $\alpha=-\beta=1 / 8, r=-1 / 8$ имеет решение $w=1+\sqrt{z}$. Полагая в формуле (8.2) $m=1 / 2, n=0$, получим решение $h=1 / 16-\sqrt{z} / 8$ уравнения (9). И обратно, по данному решению уравнения (9) с параметрами $m=1 / 2$, $n=0, r=-1 / 8$ получим с помощью формулы (8.1) решение $w=1+\sqrt{z}$ уравнения (5) с параметрами $\alpha=-\beta=1 / 8, r=-1 / 8$.

ЗАмЕчАниЕ. В работе [17] уравнение (9) содержится в формуле, определяющей общий вид дифференциального уравнения второго порядка второй степени, принадлежащего к классу уравнений $P$-типа. Однако формулы (8) взаимно однозначного соответствия между решениями уравнения (5) и уравнения (9) (позволяющие сделать вывод о принадлежности (9) к классу уравнений $P$-типа) в работе [17] не приведены.

Уравнение (9) при $m+n=0, r=1 / 2$ заменой $h \rightarrow-h$ приводится к виду

$$
z^{2} h^{\prime \prime 2}-4 h^{\prime 2}\left(z h^{\prime}-h\right)+h^{\prime}\left[\left(z-n^{2}\right) h^{\prime}-h\right]=0 .
$$

Уравнение (10) получено в работе [14] при исследовании резольвенты определителя Фредгольма интегрального оператора с ядром Бесселя. В работе [15] уравнение (10) получено как частный случай дифференциального уравнения в частных производных (уравнение для нуль-вероятностей) определителя Фредгольма интегрального уравнения с ядром Бесселя.

Теорема 1. Пусть $K(z, w), L(z, w)$ - произвольные аналитические функиии переменных $z, w$ такие, что $\partial K / \partial z \equiv \partial L / \partial w$. Тогда функиия

$$
H_{2}=H_{1}(z, u+K, w)+L
$$

определяет гамильтониан, ассочиированный с уравнением (5).

Для доказательства теоремы достаточно рассмотреть систему Гамильтона с гамильтонианом $H_{2}$, которая оказывается эквивалентной уравнению (5). 
Из теоремы 1 следует существование гамильтонианов нерационального типа, ассоциированных с уравнением (5).

Следуя работе [13], уравнение (9) будем называть $h$-уравнением. Гамильтониан $H_{1}$ будем называть простейшим рациональным гамильтонианом.

Уравнение (5) в случае $z=e^{x}, r=0$ записывается в виде эквивалентной ему автономной системы Гамильтона с гамильтонианом

$$
H_{3}(x, u, w)=e^{x} H(x, u, w),
$$

имеющей первый интеграл

$$
w(w-1)^{2} u^{2}+m(w-1)^{2} u+n w(w-1)=C,
$$

где $w=w(x), C$ - произвольная постоянная. Соотношению (11) можно придать вид

$$
\frac{w^{\prime 2}}{w(w-1)^{2}}-2 \alpha w+\frac{2 \beta}{w}=C_{1},
$$

где $C_{1}=4[C+m(m+n) / 2]$.

Наличие первого интеграла (12) уравнения (5) в случае $r=0, z=e^{x}$ и интегрируемость уравнения (12) в элементарных функциях были доказаны в работе [18].

\section{3. ГАМИЛЬТОНИАНЫ, АССОЦИИРОВАННЫЕ С УРАВНЕНИЕМ $P_{3}$}

Теорема 2. Уравнение $P_{3}$ представимо в виде системы (1) с гамильтонианом

$$
K=\frac{\alpha_{0} \lambda^{2}(u+P)^{2}}{\tau}+\frac{\beta_{0} \lambda(u+P)}{\tau}-\frac{a \lambda}{2 \alpha_{0}}+\frac{b}{2 \alpha_{0} \lambda}-\frac{c \tau \lambda^{2}}{4 \alpha_{0}}+\frac{d \tau}{4 \alpha_{0} \lambda^{2}}+Q,
$$

где $\alpha_{0} \neq 0, \beta_{0}$ - произволъные постоянные; $P(\tau, \lambda), Q(\tau, \lambda)$ - аналитические функиии, удовлетворяющие условию $\partial P / \partial \tau \equiv \partial Q / \partial \lambda$.

Для доказательства теоремы достаточно рассмотреть систему (1) с независимой переменной $\tau$ и гамильтонианом (13) и убедиться в том, что она эквивалентна уравнению $P_{3}$.

Если в формуле (13) $P=Q \equiv 0, \alpha_{0}=1, \beta_{0}=-3, a=b=0, c=1, d=-1$, то гамильтониан $K$ совпадает с $K_{0}$ с точностью до функции от $\tau$.

Гамильтониан $K_{1}$ получается из формулы (13) при $P=Q \equiv 0, \alpha_{0}=2, \beta_{0}=-3$.

Из формулы (13) следует существование гамильтонианов нерационального типа, ассоциированных с уравнением $P_{3}$. Ранее этот факт был установлен в монографии [12].

\section{4. ЗАКЛЮЧЕНИЕ}

Таким образом, в настоящей работе для уравнения $P_{5}$ в случае $\gamma \neq 0, \delta=0$ получено $h$-уравнение для простейшего ассоциированного с ним рационального гамильтониана. Получены также формулы взаимно однозначного соответствия между решениями указанных уравнений. Доказано существование ассоциированных 
с уравнением $P_{5}$ в случае $\gamma \neq 0, \delta=0$ гамильтонианов нерационального типа. Получено обобщение формул Гарнье и Окамото рациональных гамильтонианов, ассоциированных с уравнением $P_{3}$.

Другие аспекты, касающиеся ассоциированных с неприводимыми уравнениями Пенлеве гамильтонианов полиномиального типа, отражены в работах [19], [20] (см. также книгу [21]) и [22], [23].

Благодарности. Автор благодарит рецензента за проявленные интерес и внимание к работе и за конструктивные замечания.

\section{Список литературы}

[1] А.М. Бородин, Изомонодромные деформачии и уравнения Пенлеве в задачах случайно-матричного типа, Препринт ПОМИ № 04-2005; http://www.pdmi.ras.ru.

[2] M. Jimbo, T. Miwa, Y. Môry, M. Sato, Physica D, 1:1 (1980), 80-158.

[3] B. M. McCoy, T. T. Wu, C. A. Tracy, J. Math. Phys., 18:5 (1977), 1058-1092.

[4] E. Barouch, B. M. McCoy, T. T. Wu, Phys. Rev. Lett., 31:23 (1973), 1409-1411.

[5] C. A. Tracy, B. M. McCoy, Phys. Rev. Lett., 31:25 (1973), 1500-1504.

[6] T. T. Wu, B. M. McCoy, C. A. Tracy, E. Barouch, Phys. Rev. B, 13:1 (1976), 316-374.

[7] L. Onsager, Phys. Rev., 65:3-4 (1944), 117-149.

[8] М. Сато, М. Дзимбо, Т. Мива, Голономные квантовые поля, Мир, М., 1983.

[9] K. Okamoto, Proc. Japan Acad. Ser. A, 56:8 (1980), 367-371.

[10] K. Okamoto, Proc. Japan Acad. Ser. A, 56:6 (1980), 264-268.

[11] J. Malmquist, Ark. Math. Astron. Phys., 17:8 (1923), 1-89.

[12] В.В. Цегельник, Некоторые аналитические свойства и приложения решений уравнений Пенлеве-типа, БГУ, Минск, 2007.

[13] В. В. Цегельник, ТМФ, 151:1 (2007), 54-65.

[14] C. A. Tracy, H. Widom, Comm. Math. Phys., 161:2 (1994), 289-309.

[15] P. van Moerbeke, "Integrable lattices: random matrices and random permutations", Random Matrix Models and their Applications, Math. Sci. Res. Inst. Publ., 40, eds. P. Bleher,

A. Its, Cambridge Univ. Press, Cambridge, 2001, 321-406.

[16] R. Garnier, Ann. Sci.Écol Norm. Sup. (3), 29 (1912), 1-126.

[17] C. M. Cosgrove, G. Scoufis, Stud. Appl. Math., 88:1 (1993), 25-87.

[18] В. В. Голубев, Матем. сб., 28:2 (1912), 323-349.

[19] S. Yu. Slavyanov, J. Phys. A, 29:22 (1996), 7329-7335.

[20] С. Ю. Славянов, ТМФ, 123:3 (2000), 395-406.

[21] С. Славянов, В. Лай, Специальные функиии: единая теория, основанная на анализе особенностей, Невский диалект, С.-Пб., 2002.

[22] Б. И. Сулейманов, ТМФ, 156:3 (2008), 364-377.

[23] K. Iwasaki, H. Kimura, S. Shimomura, M. Yoshida, From Gauss to Painlevé: a Modern Theory of Special Functions, Aspects Math., E16, Vieweg, Braunschweig, Germany, 1991.

Поступила в редакцию 26.12.2008, после доработки 25.05.2009 